Acta Bot. Croat. 71 (2), 229-248, 2012

\title{
Floristic analysis of a high-speed railway embankment in a Mediterranean landscape
}

\author{
Goffredo Filibeck $^{1 *}$, Paolo Cornelini ${ }^{2 * *}$, Paolo Petrella $^{3}$ \\ ${ }^{1}$ Department of Agriculture, Forests, Nature and Energy, University of Tuscia, I-01100 \\ Viterbo, Italy \\ ${ }^{2}$ Istituto Sperimentale Ferrovie dello Stato, Roma, Italy \\ ${ }^{3}$ Via Tuscolana 909, I-00174 Roma, Italy
}

\begin{abstract}
We analyzed the floristic composition of a $4.5 \mathrm{~km}$-long segment of a high-speed railway in Lazio, central Italy, which travels on an artificial embankment through an intensively-farmed landscape. In total, 287 vascular plant species were recorded. The life-form distribution was found to be similar to that of the regional species pool, with high percentages of therophytes (38\%) and phanerophytes (13\%). In the chorological spectrum the Mediterranean floristic element prevailed (44\%), while alien species were $8 \%$ of the flora. The phytosociological spectrum showed a high diversity of characteristic species from the class Stellarietea mediae or its subordinate syntaxa (26\%), and in particular from the order Thero-Brometalia (Mediterranean, sub-nitrophilous annual communities). Species from forest syntaxa had a relatively high diversity $(9 \%)$. These results suggest that the ecological filtering provided by the Mediterranean regional climate controlled species assemblage even in a completely artificial habitat, preventing floristic homogenization: the flora of the studied railway section is only partially »ruderalized «, while it keeps strong links with the regional (semi-) natural plant communities. However, in contrast to what is observed in central and north Europe, the railway sides studied in the present paper do not seem to represent a refugial habitat for rare species from grassland communities, mainly because in Italy semi-natural dry grasslands are still widely represented.
\end{abstract}

Key words: anthropogenic, habitat, artificial soil, life-form, railway flora, Lazio, Italy

\section{Introduction}

Railways and railway verges are colonized by a high number of plant species, and have been the subject of botanical studies for more than 150 years (see references in: MÜHLENBACH

* Corresponding author, e-mail: filibeck@unitus.it

** Present address: Via Scandriglia 7, I-00199 Roma, Italy

Copyright ${ }^{\circledR} 2012$ by Acta Botanica Croatica, the Faculty of Science, University of Zagreb. All rights reserved. 
1979; Brandes 1983, 1993, 2008; Cornelini and Petrella 1996; SchoenenBerger et al. 2002; NOWIŃSKA and CZARNA 2008).

Some works have focused on the negative role of rail lines as dispersal pathways for alien species (Schoenenberger et al. 2002, Christen and Matlack 2006, Ranta 2008, TRET'YAKOVA 2010) - although due to the decrease in grain and livestock rail transportation in the last few decades, railways have nowadays a less rich alien flora than observed in the first half of the 20th century (BRANDES 1983: 38, CoRnElini and PETRELla 1996, TinNeR and SCHUMACHER 2004).

On the other hand, several central and north European studies have underlined the role of road and railway verges and embankments in hosting a surprisingly high percentage of a country's native floristic diversity, and have showed that they can even act as habitats for rare or endangered species (e.g. SARGENT 1984, BRANDES 1993, TikKA et al. 2000, SchAFFERS and SyKora 2002, WitTig 2002, TinNER and Schumacher 2004, RANTA 2008). A floristic survey of the Dutch railway network found a total of 1,026 species, i.e. $63 \%$ of the country's flora (KOSTER 1987), while in Britain a total of 1,632 vascular plant species and subspecies was recorded from railway land (SARGENT 1984). In Finland, $4 \%$ of the nationally endangered species regularly occur on road and rail verges (TIKKA et al. 2000).

In natural landscapes, roads and railways mainly function as a source of fragmentation for habitats and populations (CHRISTEN and MATLACK 2006, FisCHER and LinDENMAYER 2007). In highly transformed landscapes, on the other hand, rail and road sides - being a relatively undisturbed habitat compared to the surrounding areas (MESSENGER 1968, BRANDES 1993) may act as corridors for the survival and dispersal of plant populations from endangered natural or semi-natural communities (TIKKA et al. 2001, RANTA 2008), especially if the railway verges are managed according to biodiversity-oriented criteria (PARR and WAY 1988; CORNELINI et al. 1990; CORNELINI 1994a, b; TIKKA et al. 2000, 2001).

However, in Italy (and, to our knowledge, in most of Mediterranean Europe) botanical studies on rail lines are much scarcer than in central European countries (cf. BRANDES 1993: 441) and are focused on railway stations only (CACCIATO 1952; CoRNELINI and Petrella 1996, 1997) or on the classification of plant communities (BRANDES 1992; CORNELINI 1994a, b).

In this study, we present the results of a complete floristic survey of a $4.5 \mathrm{~km}$ segment of a high-speed railway (i.e. a passenger rail transport system that operates above $200 \mathrm{~km} \mathrm{~h}^{-1}$ ), across an intensively farmed landscape within the Mediterranean region of Central Italy.

The rail line segment was built in 1975; the floristic survey took place in 1993, within a project carried out by one of the authors (P. C.) at the Research Institute of Italian State Railways, aimed at monitoring the plant-cover evolution of rail embankments without hydroseeding or other revegetation practices. The results of the floristic survey remained unpublished. We believe they are nowadays of interest, for many reasons. First, no other complete floristic studies have been performed, to our knowledge, on a long segment of an Italian railway line beyond railway stations and urban areas; second, it is now very difficult to obtain permission from the railway authorities to perform botanical studies on high-speed lines, because of increased operational speeds and the stricter security policy; finally, recently enforced maintenance procedures now include much more frequent mowing of embankments than at the time of our survey, so that the data collected under a lower disturbance regime can be useful for further studies comparing the effects of different management practices. 


\section{Materials and methods}

The surveyed railway segment is $4.5 \mathrm{~km}$ long and belongs to the high-speed Florence-Rome line. This line was the first high-speed railway opened in Europe (it started operating in 1977).

The studied section is located in the Municipality of Rome (Lazio, central Italy), and travels on an uninterrupted embankment, i.e. a raised earth and gravel structure (with the exception of a bridge over the river Tiber), thus providing with its sides a large surface of a man-made substrate, raising above the cultivated land. The embankment begins at the end of a viaduct near Settebagni (at the $18 \mathrm{~km}$ milepost), and ends at the 22,5 milepost (where a series of tunnels begins). It was built in 1975 and is made up of a mixture of coarse-grained soil and crushed rock that was compacted during the construction work so as to ensure mechanical resistance and low permeability. In contrast to the current usual practice in analogous earthworks, the slopes of the embankment were not sown with stabilizing grass mixtures during or after construction, so the colonizing process was completely spontaneous. Ever since the line came into operation, herbicide spraying has been done on the track ballast only; the embankment slopes, at the time of the field study, were left mostly undisturbed.

The dominant vegetation types on the embankment slopes and sides included: annual Mediterranean grasslands, patches of perennial grasslands, hygrophilous herbaceous communities (along the drainage ditches), stands of shrubs (mostly dominated by Spartium junceum) (cf. CORNELINI 1994b).

The rail segment travels in the Tiber plain, at approx. $20 \mathrm{~m}$ a.s.1., with a N-S orientation; the railway is more or less parallel to the riverbed, although due to the many meanders the distance between the rail line and the river banks varies between ca. 100-1000 m (except where the railway crosses the river). The plain is ca $3 \mathrm{~km}$ wide and is surrounded by a series of pyroclastic hills (max. altitude $150 \mathrm{~m}$ ). The climate is Mediterranean; namely, the area belongs to the meso-Mediterranean, sub-humid belt, i.e. a climate type with an annual rainfall of approx. 800-900 mm, a marked summer drought from June to August and mild winter temperatures (BLASI 1994).

Much of the floodplain surrounding the rail segment is intensively farmed: hedgerows and other residual habitats for wild plants are scarce. The river banks host poorly-preserved fragments of riparian vegetation, dominated by Populus sp.pl. and Salix sp. pl. The heavily-trafficked Milan-Rome motorway, too, travels in the floodplain parallel to the rail line, at a distance of a few hundred meters. The hill-slopes feature deciduous, xerophilous coppices dominated by Quercus cerris (Teucrio siculi-Quercion cerridis alliance) (BLASI et al. 2010, FILIBECK and SCOPPOLA 2011).

The floristic survey was done in 1993. The whole length of the segment of the railway was walked, on both sides of the embankment, in both directions. The walk was repeated in different seasons. All vascular plant species observed on the embankment slopes, including the drainage ditches at the base, were recorded. The survey area defined in this way was ca $20 \mathrm{~m}$ wide on each side of the rail line. The surveyed area did not include the ballast, because on high-speed lines the permanent way is kept free of vascular plants through frequent herbicide spraying. The plant list of the first 1,000 $\mathrm{m}$ was recorded separately, in order to compare the species richness of a 1-km segment with that of the whole $4.5 \mathrm{~km}$ stretch. It was not possible (due to the inherent safety problems of working on a high-speed line) to divide the study site into statistically formalized sampling units (plots or transects) in order to obtain 
quantitative data on species abundance. However, 12 phytosociological relevés were done on the embankment slopes at the beginning of the field study (and published as a synoptic table in CORNELINI 1994b). No significant differences were observed, during the phytosociological survey between the east- and west-facing slopes of the embankment: therefore, during the subsequent floristic survey no separate floristic lists were recorded for the two sides.

Identification of species and nomenclature followed PIGNATTI (1982). Each species was then assigned a life-form type and a geographic element according to PigNATTI (1982), in order to analyze the relationships with the regional species-pool. The subdivision of floristic zones and regions, and thus of geographic elements, used by PIGNATTI (1982), stresses some patterns relevant for the Italian peninsula (e.g. a distinction between »steno-Mediterranean « and »euri-Mediterranean« species is adopted) - thus it does not match the other systems used in central Europe perfectly.

A species was defined as »alien« if included in the check-list of Italian alien taxa by CELESTI-GRAPOW et al. (2010), and its status (casual, naturalized or invasive) was taken from the same source. Alien »species« defined in this way include also taxa originating in cultivation.

Finally, each species was assigned to a phytosociological unit, in order to analyze the relationships between the floristic assemblage of the railway sides and the (semi-)natural vegetation types of the surrounding landscape. In order to do so in a standardized and repeatable way, this was done assigning to each phytosociological class or order only those plants that are considered »characteristic species « of that unit, following reference manuals (OBERDORFER 1992, MuCiNA et al. 1993) and syntaxonomical revisions (IzCO 1977, BIONDI et al. 1995, BLASI et al. 2004, Gigante and VENANZONI 2007).

\section{Results}

A total of 287 vascular plant species was recorded in the research area (Tab.1); 207 species (72\%) were found within the first $1,000 \mathrm{~m}$. The richest family was found to be Asteraceae (16.4\%), followed by Fabaceae and Poaceae (ca. 12\%).

The life-form types with the highest diversity were therophytes and hemicryptophytes accounting for $38 \%$ each (Fig. 1). As for hemicryptophytes, many of the species found in the

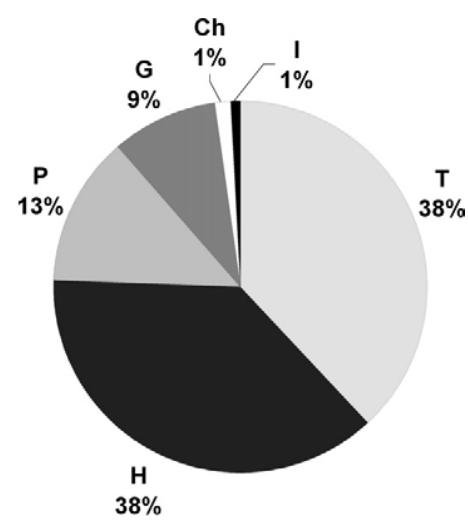

Fig. 1. Life-form distribution in the flora of the railway segment. For abbreviations see table 1. 
Tab. 1. List of recorded species. Legend of abbreviations - Life forms: $\mathbf{C h}$ - chamaephytes, $\mathbf{G}-$ geophytes, $\mathbf{H}$ - hemicryptophytes, H(b) - biennial hemicryptophytes, I - hydrophytes, $\mathbf{P}$ phanerophytes, $\mathbf{T}$ - therophytes. Geographic elements: »Circumboreal« includes also the Euro-Siberian element; »Cosmopolitan « includes also the Sub-Cosmopolitan element; »Eurasiatic « includes also the European element. Origin of aliens: Afr - Africa, Eur - Europe, hort. - horticultural (taxa originated in cultivation), N Amer - North America, S Amer South or Central America. Status of aliens in Lazio: cas - casual, inv - invasive, nat naturalized. Vegetation units: Av-Artemisietea vulgaris, C-M -Cisto-Micromerietea, $\mathbf{F}-\mathbf{B}$ Festuco-Brometea, G-U - Galio-Urticetea, $\mathbf{H g}$ - Helianthemetea guttati, hy - riparian and hygrophilous communities, M-A - Molinio-Arrhenateretea, Qi - Quercetea ilicis, Q-F Querco-Fagetea, R-P - Rhamno-Prunetea, $\mathbf{S t}$ - Stellarietea mediae (except Thero-Brometalia),T-Brom - Thero-Brometalia, T-G - Trifolio-Geranietea.

\begin{tabular}{|c|c|c|c|c|c|}
\hline Species & $\begin{array}{l}\text { Life } \\
\text { form }\end{array}$ & $\begin{array}{l}\text { Geographic } \\
\text { element }\end{array}$ & $\begin{array}{l}\text { Origin } \\
\text { of aliens }\end{array}$ & $\begin{array}{l}\text { Status } \\
\text { of aliens } \\
\text { (Lazio) }\end{array}$ & $\begin{array}{l}\text { Vegeta- } \\
\text { tion } \\
\text { units }\end{array}$ \\
\hline Acer negundo L. & $\mathrm{P}$ & Alien & N Amer & cas & \\
\hline Agrimonia eupatoria $\mathrm{L}$. & $\mathrm{H}$ & Cosmopolitan & & & $\mathrm{T}-\mathrm{G}$ \\
\hline Agropyron repens (L.) Beauv. & G & Circumboreal & & & Av \\
\hline Ailanthus altissima (Miller) Swingle & $\mathrm{P}$ & Alien & Asia & inv & \\
\hline Alcea setosa (Boiss.) Alef. & $\mathrm{H}$ & Alien & Eur & nat & \\
\hline Alisma plantago-aquatica $\mathrm{L}$. & I & Cosmopolitan & & & hy \\
\hline Allium ampeloprasum $\mathrm{L}$. & G & Euri-Mediterranean & & & \\
\hline Alopecurus myosuroides Hudson & $\mathrm{T}$ & Cosmopolitan & & & St \\
\hline Althaea cannabina $\mathrm{L}$. & $\mathrm{H}$ & Eurasiatic & & & \\
\hline Amaranthus retroflexus $\mathrm{L}$. & $\mathrm{T}$ & Alien & N Amer & inv & \\
\hline Anagallis arvensis $\mathrm{L}$. & $\mathrm{T}$ & Euri-Mediterranean & & & St \\
\hline Anchusa hybrida Ten. & $\mathrm{H}$ & Steno-Mediterranean & & & \\
\hline Anchusa italica Retz. & $\mathrm{H}$ & Euri-Mediterranean & & & \\
\hline Andryala integrifolia $\mathrm{L}$. & $\mathrm{T}$ & Euri-Mediterranean & & & St \\
\hline Anthemis arvensis $\mathrm{L}$. & $\mathrm{T}$ & Steno-Mediterranean & & & St \\
\hline Anthemis tinctoria $\mathrm{L}$. & $\mathrm{H}(\mathrm{b})$ & Eurasiatic & & & Av \\
\hline Apium nodiflorum (L.) Lag. & $\mathrm{H}$ & Euri-Mediterranean & & & hy \\
\hline Arenaria serpyllifolia $\mathrm{L}$. & $\mathrm{T}$ & Cosmopolitan & & & $\mathrm{Hg}$ \\
\hline Artemisia verlotorum Lamotte & $\mathrm{H}$ & Alien & Asia & inv & \\
\hline Artemisia vulgaris $\mathrm{L}$. & $\mathrm{H}$ & Circumboreal & & & Av \\
\hline Arum italicum Miller & G & Steno-Mediterranean & & & G-U \\
\hline Arundo donax $\mathrm{L}$. & G & Alien & Asia & inv & \\
\hline Asparagus acutifolius $\mathrm{L}$. & $\mathrm{P}$ & Steno-Mediterranean & & & Qi \\
\hline Astragalus hamosus $\mathrm{L}$. & $\mathrm{T}$ & Medit.-Turanic & & & T-Brom \\
\hline Avena barbata Potter & $\mathrm{T}$ & Euri-Mediterranean & & & T-Brom \\
\hline Avena fatua $\mathrm{L}$. & $\mathrm{T}$ & Eurasiatic & & & St \\
\hline Avena sterilis $\mathrm{L}$. & $\mathrm{T}$ & Euri-Mediterranean & & & T-Brom \\
\hline Bellevalia romana (L.) Sweet & G & Euri-Mediterranean & & & \\
\hline Bellis perennis $\mathrm{L}$. & $\mathrm{H}$ & Eurasiatic & & & M-A \\
\hline Beta vulgaris L. cv. & $\mathrm{H}$ & Alien & hort. & cas & \\
\hline Blackstonia perfoliata (L.) Hudson & $\mathrm{T}$ & Euri-Mediterranean & & & $\mathrm{Hg}$ \\
\hline Borago officinalis L. & $\mathrm{T}$ & Euri-Mediterranean & & & \\
\hline Briza maxima $\mathrm{L}$. & $\mathrm{T}$ & Subtropical & & & $\mathrm{Hg}$ \\
\hline
\end{tabular}


Filibeck G., Cornelini P., Petrella P.

Tab. 1. - continued

\begin{tabular}{|c|c|c|c|c|c|}
\hline Species & $\begin{array}{l}\text { Life } \\
\text { form }\end{array}$ & $\begin{array}{l}\text { Geographic } \\
\text { element }\end{array}$ & $\begin{array}{l}\text { Origin } \\
\text { of aliens }\end{array}$ & $\begin{array}{l}\text { Status } \\
\text { of aliens } \\
\text { (Lazio) }\end{array}$ & $\begin{array}{l}\text { Vegeta- } \\
\text { tion } \\
\text { units }\end{array}$ \\
\hline Bromus gussonei Parl. & $\mathrm{T}$ & Euri-Mediterranean & & & $\mathrm{St}$ \\
\hline Bromus hordeaceus L. & $\mathrm{T}$ & Cosmopolitan & & & T-Brom \\
\hline Bromus madritensis $\mathrm{L}$. & $\mathrm{T}$ & Euri-Mediterranean & & & T-Brom \\
\hline Bromus rigidus Roth & $\mathrm{T}$ & Subtropical & & & T-Brom \\
\hline Broussonetia papyrifera (L.) Vent. & $\mathrm{P}$ & Alien & Asia & inv & \\
\hline Calamintha nepeta (L.) Savi & $\mathrm{H}$ & Montane-Mediterranean & & & \\
\hline Calendula arvensis $\mathrm{L}$. & $\mathrm{T}$ & Euri-Mediterranean & & & St \\
\hline Calystegia sepium (L.) R.Br. & $\mathrm{H}$ & Eurasiatic & & & G-U \\
\hline Campanula rapunculus $\mathrm{L}$. & $\mathrm{H}(\mathrm{b})$ & Eurasiatic & & & \\
\hline Capsella bursa pastoris (L.) & $\mathrm{H}(\mathrm{b})$ & Cosmopolitan & & & St \\
\hline Cardamine hirsuta $\mathrm{L}$. & $\mathrm{T}$ & Cosmopolitan & & & \\
\hline Cardaria draba (L.) Desv. & G & Medit.-Turanic & & & \\
\hline Carduus nutans $\mathrm{L}$. & $\mathrm{H}(\mathrm{b})$ & Atlantic & & & \\
\hline Carduus pycnocephalus $\mathrm{L}$. & $\mathrm{H}(\mathrm{b})$ & Medit.-Turanic & & & St \\
\hline Carex caryophyllea La Tourr. & $\mathrm{H}$ & Eurasiatic & & & F-B \\
\hline Carex divisa Hudson & G & Atlantic & & & \\
\hline Carex divulsa Stokes & $\mathrm{H}$ & Euri-Mediterranean & & & \\
\hline Carex flacca Schreber & G & Eurasiatic & & & \\
\hline Carex otrubae Podp. & $\mathrm{H}$ & Atlantic & & & hy \\
\hline Carlina corymbosa $\mathrm{L}$. & $\mathrm{H}$ & Steno-Mediterranean & & & $\mathrm{Hg}$ \\
\hline Celtis australis $\mathrm{L}$. & $\mathrm{P}$ & Euri-Mediterranean & & & \\
\hline Centaurea solstitialis $\mathrm{L}$. & $\mathrm{H}(\mathrm{b})$ & Steno-Mediterranean & & & Av \\
\hline Centaurium erythraea Rafn & $\mathrm{H}(\mathrm{b})$ & Eurasiatic & & & F-B \\
\hline Cephalaria transsylvanica (L.) Schrader & $\mathrm{T}$ & Eurasiatic & & & \\
\hline Cerastium glomeratum Thuill. & $\mathrm{T}$ & Euri-Mediterranean & & & St \\
\hline Cercis siliquastrum $\mathrm{L}$. & $\mathrm{P}$ & Eurasiatic & & & Q-F \\
\hline Chenopodium album $\mathrm{L}$. & $\mathrm{T}$ & Cosmopolitan & & & St \\
\hline Chondrilla juncea $\mathrm{L}$. & $\mathrm{H}$ & Eurasiatic & & & \\
\hline Chrozophora tinctoria (L.) Juss. & $\mathrm{T}$ & Medit.-Turanic & & & \\
\hline Cichorium intybus $\mathrm{L}$. & $\mathrm{H}$ & Eurasiatic & & & \\
\hline Cirsium arvense (L.) Scop. & G & Eurasiatic & & & $\mathrm{St}$ \\
\hline Cirsium vulgare (Savi) Ten. & $\mathrm{H}(\mathrm{b})$ & Eurasiatic & & & Av \\
\hline Clematis vitalba $\mathrm{L}$. & $P$ & Eurasiatic & & & Q-F \\
\hline Coleostephus myconis (L.) Cass. & $\mathrm{T}$ & Steno-Mediterranean & & & \\
\hline Convolvulus arvensis $\mathrm{L}$. & G & Eurasiatic & & & Av \\
\hline Convolvulus cantabrica $\mathrm{L}$. & $\mathrm{H}$ & Euri-Mediterranean & & & $\mathrm{Hg}$ \\
\hline Conyza bonariensis (L.) Cronq. & $\mathrm{T}$ & Alien & S Amer & inv & \\
\hline Conyza canadensis (L.) Cronq. & $\mathrm{T}$ & Alien & N Amer & inv & \\
\hline Cornus sanguinea $\mathrm{L}$. & $\mathrm{P}$ & Eurasiatic & & & R-P \\
\hline Crataegus monogyna Jacq. & $P$ & Eurasiatic & & & R-P \\
\hline Crepis bursifolia $\mathrm{L}$. & $\mathrm{H}$ & Endem. & & & \\
\hline Crepis neglecta $\mathrm{L}$. & $\mathrm{T}$ & Euri-Mediterranean & & & $\mathrm{Hg}$ \\
\hline Crepis sancta (L.) Babc. & $\mathrm{T}$ & Medit.-Turanic & & & St \\
\hline
\end{tabular}


Tab. 1. - continued

\begin{tabular}{|c|c|c|c|c|c|}
\hline Species & $\begin{array}{l}\text { Life } \\
\text { form }\end{array}$ & $\begin{array}{l}\text { Geographic } \\
\text { element }\end{array}$ & $\begin{array}{l}\text { Origin } \\
\text { of aliens }\end{array}$ & $\begin{array}{l}\text { Status } \\
\text { of aliens } \\
\text { (Lazio) }\end{array}$ & $\begin{array}{l}\text { Vegeta- } \\
\text { tion } \\
\text { units }\end{array}$ \\
\hline Crepis vesicaria $\mathrm{L}$. & $\mathrm{T}$ & Atlantic & & & St \\
\hline Cruciata laevipes Opiz & $\mathrm{H}$ & Eurasiatic & & & G-U \\
\hline Cyclamen hederifolium Aiton & $\mathrm{G}$ & Steno-Mediterranean & & & Q-F \\
\hline Cynara cardunculus $\mathrm{L}$. & $\mathrm{H}$ & Steno-Mediterranean & & & \\
\hline Cynodon dactylon (L.) Pers. & $\mathrm{G}$ & Cosmopolitan & & & \\
\hline Cyperus longus $\mathrm{L}$. & G & Eurasiatic & & & hy \\
\hline Cyperus rotundus $\mathrm{L}$. & G & Cosmopolitan & & & hy \\
\hline Dactylis glomerata $\mathrm{L}$. & $\mathrm{H}$ & Eurasiatic & & & M-A \\
\hline Dasypirum villosum (L.) Borbas & $\mathrm{T}$ & Medit.-Turanic & & & T-Brom \\
\hline Daucus carota $\mathrm{L}$. & $\mathrm{H}(\mathrm{b})$ & Eurasiatic & & & Av \\
\hline Diplotaxis tenuifolia (L.) Dc. & $\mathrm{H}$ & Atlantic & & & \\
\hline Dipsacus fullonum $\mathrm{L}$. & $\mathrm{H}(\mathrm{b})$ & Euri-Mediterranean & & & $\mathrm{Av}$ \\
\hline Dorycnium hirsutum (L.) Ser. & $\mathrm{Ch}$ & Euri-Mediterranean & & & \\
\hline Ecballium elaterium (L.) A.Rich. & G & Euri-Mediterranean & & & St \\
\hline Echium italicum $\mathrm{L}$. & $\mathrm{H}(\mathrm{b})$ & Euri-Mediterranean & & & $\mathrm{Av}$ \\
\hline Echium plantagineum $\mathrm{L}$. & $\mathrm{T}$ & Euri-Mediterranean & & & T-Brom \\
\hline Echium vulgare $\mathrm{L}$. & $\mathrm{H}(\mathrm{b})$ & Eurasiatic & & & F-B \\
\hline Epilobium hirsutum $\mathrm{L}$. & $\mathrm{H}$ & Eurasiatic & & & \\
\hline Epilobium tetragonum $\mathrm{L}$. & $\mathrm{H}$ & Eurasiatic & & & \\
\hline Equisetum arvense $\mathrm{L}$. & G & Circumboreal & & & hy \\
\hline Equisetum ramosissimum Desf. & G & Circumboreal & & & hy \\
\hline Erodium malacoides (L.) L'Her. & $\mathrm{T}$ & Steno-Mediterranean & & & St \\
\hline Eryngium campestre $\mathrm{L}$. & $\mathrm{H}$ & Euri-Mediterranean & & & F-B \\
\hline Euonymus europaeus $\mathrm{L}$. & $\mathrm{P}$ & Eurasiatic & & & R-P \\
\hline Eupatorium cannabinum $\mathrm{L}$. & $\mathrm{H}$ & Eurasiatic & & & hy \\
\hline Euphorbia falcata $\mathrm{L}$. & $\mathrm{T}$ & Euri-Mediterranean & & & $\mathrm{Hg}$ \\
\hline Euphorbia helioscopia L. & $\mathrm{T}$ & Cosmopolitan & & & St \\
\hline Euphorbia platyphyllos L. & $\mathrm{T}$ & Euri-Mediterranean & & & \\
\hline Euphorbia prostrata Aiton & $\mathrm{T}$ & Alien & S Amer & inv & \\
\hline Euphorbia segetalis L. & $\mathrm{T}$ & Steno-Mediterranean & & & \\
\hline Fallopia dumetorum (L.) Holub & $\mathrm{T}$ & Circumboreal & & & \\
\hline Ferula communis $\mathrm{L}$. & $\mathrm{H}$ & Euri-Mediterranean & & & \\
\hline Festuca arundinacea Schreber & $\mathrm{H}$ & Eurasiatic & & & \\
\hline Ficus carica $\mathrm{L}$. & $\mathrm{P}$ & Medit.-Turanic & & & \\
\hline Foeniculum vulgare Miller & $\mathrm{H}$ & Euri-Mediterranean & & & T-Brom \\
\hline Fraxinus ornus $\mathrm{L}$. & $\mathrm{P}$ & Eurasiatic & & & Q-F \\
\hline Fraxinus oxycarpa Bieb. & $\mathrm{P}$ & Eurasiatic & & & hy \\
\hline Fumaria capreolata $\mathrm{L}$. & $\mathrm{T}$ & Euri-Mediterranean & & & St \\
\hline Fumaria officinalis L. & $\mathrm{T}$ & Eurasiatic & & & St \\
\hline Galactites tomentosa Moench & $\mathrm{H}(\mathrm{b})$ & Steno-Mediterranean & & & T-Brom \\
\hline Galega officinalis L. & $\mathrm{H}$ & Eurasiatic & & & G-U \\
\hline Galium album Miller & $\mathrm{H}$ & Eurasiatic & & & F-B \\
\hline Galium aparine $\mathrm{L}$. & $\mathrm{T}$ & Eurasiatic & & & $\mathrm{G}-\mathrm{U}$ \\
\hline
\end{tabular}


Filibeck G., Cornelini P., Petrella P.

Tab. 1. - continued

\begin{tabular}{|c|c|c|c|c|c|}
\hline Species & $\begin{array}{l}\text { Life } \\
\text { form }\end{array}$ & $\begin{array}{l}\text { Geographic } \\
\text { element }\end{array}$ & $\begin{array}{l}\text { Origin } \\
\text { of aliens }\end{array}$ & $\begin{array}{l}\text { Status } \\
\text { of aliens } \\
\text { (Lazio) }\end{array}$ & $\begin{array}{l}\text { Vegeta- } \\
\text { tion } \\
\text { units }\end{array}$ \\
\hline Gastridium ventricosum (Gouan) Sch. et Th. & $\mathrm{T}$ & Atlantic & & & T-Brom \\
\hline Geranium dissectum $\mathrm{L}$. & $\mathrm{T}$ & Eurasiatic & & & St \\
\hline Geranium molle $\mathrm{L}$. & $\mathrm{T}$ & Eurasiatic & & & St \\
\hline Geranium robertianum $\mathrm{L}$. & $\mathrm{T}$ & Cosmopolitan & & & St \\
\hline Hedera helix $\mathrm{L}$. & $\mathrm{P}$ & Euri-Mediterranean & & & Q-F \\
\hline Hedysarum coronarium $\mathrm{L}$. & $\mathrm{H}$ & Steno-Mediterranean & & & \\
\hline Heliotropium europaeum $\mathrm{L}$. & $\mathrm{T}$ & Euri-Mediterranean & & & St \\
\hline Holcus lanatus L. & $\mathrm{H}$ & Circumboreal & & & M-A \\
\hline Hypericum perforatum $\mathrm{L}$. & $\mathrm{H}$ & Eurasiatic & & & T-G \\
\hline Hypochoeris achyrophorus $\mathrm{L}$. & $\mathrm{T}$ & Steno-Mediterranean & & & $\mathrm{Hg}$ \\
\hline Hypochoeris glabra $\mathrm{L}$. & $\mathrm{T}$ & Euri-Mediterranean & & & \\
\hline Hypochoeris radicata $\mathrm{L}$. & $\mathrm{H}$ & Eurasiatic & & & F-B \\
\hline Inula conyza $\mathrm{DC}$. & $\mathrm{H}(\mathrm{b})$ & Eurasiatic & & & T-G \\
\hline Inula viscosa (L.) Aiton & $\mathrm{H}$ & Euri-Mediterranean & & & \\
\hline Iris germanica $\mathrm{L}$. & G & Alien & hort. & nat & \\
\hline Juglans regia $\mathrm{L}$. & $\mathrm{P}$ & Alien & Asia & cas & \\
\hline Knautia integrifolia (L.) Bertol. & $\mathrm{T}$ & Euri-Mediterranean & & & St \\
\hline Lactuca serriola $\mathrm{L}$. & $\mathrm{H}(\mathrm{b})$ & Eurasiatic & & & \\
\hline Lathyrus clymenum L. & $\mathrm{T}$ & Steno-Mediterranean & & & St \\
\hline Lathyrus pratensis L. & $\mathrm{H}$ & Eurasiatic & & & M-A \\
\hline Lathyrus sylvestris $\mathrm{L}$. & $\mathrm{H}$ & Eurasiatic & & & T-G \\
\hline Laurus nobilis L. & $\mathrm{P}$ & Steno-Mediterranean & & & Qi \\
\hline Lavatera punctata All. & $\mathrm{T}$ & Steno-Mediterranean & & & \\
\hline Lemna minor $\mathrm{L}$. & I & Cosmopolitan & & & hy \\
\hline Linaria vulgaris Miller & $\mathrm{H}$ & Eurasiatic & & & $\mathrm{Av}$ \\
\hline Linum bienne Miller & $\mathrm{H}(\mathrm{b})$ & Euri-Mediterranean & & & F-B \\
\hline Lophochloa cristata (L.) Hyl. & $\mathrm{T}$ & Cosmopolitan & & & \\
\hline Lotus corniculatus $\mathrm{L}$. & $\mathrm{H}$ & Eurasiatic & & & T-G \\
\hline Lotus ornithopodioides L. & $\mathrm{T}$ & Steno-Mediterranean & & & T-Brom \\
\hline Lycopus europaeus $\mathrm{L}$. & $\mathrm{H}$ & Eurasiatic & & & hy \\
\hline Malus domestica Borkh. & $\mathrm{P}$ & Alien & hort. & cas & \\
\hline Malus sylvestris Miller & $\mathrm{P}$ & Eurasiatic & & & Q-F \\
\hline Malva sylvestris $\mathrm{L}$. & $\mathrm{H}$ & Circumboreal & & & $\mathrm{Av}$ \\
\hline Medicago lupulina $\mathrm{L}$. & $\mathrm{T}$ & Eurasiatic & & & St \\
\hline Medicago orbicularis (L.) Bartal. & $\mathrm{T}$ & Euri-Mediterranean & & & T-Brom \\
\hline Medicago sativa $\mathrm{L}$. & $\mathrm{H}$ & Eurasiatic & & & St \\
\hline Melica ciliata $\mathrm{L}$. & $\mathrm{H}$ & Euri-Mediterranean & & & F-B \\
\hline Melilotus alba Medicus & $\mathrm{T}$ & Eurasiatic & & & \\
\hline Melilotus indica (L.) All. & $\mathrm{T}$ & Medit.-Turanic & & & \\
\hline Melilotus officinalis (L.) Pallas & $\mathrm{H}(\mathrm{b})$ & Eurasiatic & & & \\
\hline Melissa romana Miller & $\mathrm{H}$ & Steno-Mediterranean & & & \\
\hline Mentha suaveolens Ehrh. & $\mathrm{H}$ & Euri-Mediterranean & & & \\
\hline Mercurialis аппиа L. & $\mathrm{T}$ & Eurasiatic & & & St \\
\hline
\end{tabular}


Tab. 1. - continued

\begin{tabular}{|c|c|c|c|c|c|}
\hline Species & $\begin{array}{l}\text { Life } \\
\text { form }\end{array}$ & $\begin{array}{l}\text { Geographic } \\
\text { element }\end{array}$ & $\begin{array}{l}\text { Origin } \\
\text { of aliens }\end{array}$ & $\begin{array}{l}\text { Status } \\
\text { of aliens } \\
\text { (Lazio) }\end{array}$ & $\begin{array}{l}\text { Vegeta- } \\
\text { tion } \\
\text { units }\end{array}$ \\
\hline Micromeria graeca $($ L.) Bentham & $\mathrm{Ch}$ & Steno-Mediterranean & & & C-M \\
\hline Myosotis arvensis (L.) Hill & $\mathrm{T}$ & Eurasiatic & & & St \\
\hline Narcissus tazetta $\mathrm{L}$. & G & Steno-Mediterranean & & & \\
\hline Nigella damascena $\mathrm{L}$. & $\mathrm{T}$ & Euri-Mediterranean & & & $\mathrm{Hg}$ \\
\hline Odontites rubra (Baumg.) Opiz & $\mathrm{T}$ & Eurasiatic & & & \\
\hline Onobrychis viciifolia Scop. & $\mathrm{H}$ & Montane-Mediterranean & & & F-B \\
\hline Ononis spinosa $\mathrm{L}$. & $\mathrm{Ch}$ & Euri-Mediterranean & & & F-B \\
\hline Onopordum illyricum $\mathrm{L}$. & $\mathrm{H}(\mathrm{b})$ & Steno-Mediterranean & & & Av \\
\hline Ophrys apifera Hudson & G & Euri-Mediterranean & & & F-B \\
\hline Origanum vulgare $\mathrm{L}$. & $\mathrm{H}$ & Eurasiatic & & & $\mathrm{T}-\mathrm{G}$ \\
\hline Ornithogalum umbellatum $\mathrm{L}$. & G & Euri-Mediterranean & & & St \\
\hline Oryzopsis miliacea (L.) Asch. et Schweinf. & $\mathrm{H}$ & Steno-Mediterranean & & & \\
\hline Oxalis corniculata $\mathrm{L}$. & $\mathrm{H}$ & Euri-Mediterranean & & & \\
\hline Oxalis dillenii Jacq. & $\mathrm{H}$ & Alien & N Amer & inv & \\
\hline Pallenis spinosa (L.) Cass. & $\mathrm{T}$ & Euri-Mediterranean & & & \\
\hline Papaver dubium $\mathrm{L}$. & $\mathrm{T}$ & Medit.-Turanic & & & \\
\hline Papaver rhoeas $\mathrm{L}$. & $\mathrm{T}$ & Medit.-Turanic & & & St \\
\hline Parentucellia viscosa (L.) Caruel & $\mathrm{T}$ & Atlantic & & & \\
\hline Parietaria diffusa $\mathrm{M}$. et $\mathrm{K}$. & $\mathrm{H}$ & Euri-Mediterranean & & & \\
\hline Parthenocissus quinquefolia (L.) Planchon & $\mathrm{P}$ & Alien & N Amer & nat & \\
\hline Petrorhagia prolifera (L.) Ball et Heyw. & $\mathrm{T}$ & Euri-Mediterranean & & & \\
\hline Phalaris bulbosa $\mathrm{L}$. & $\mathrm{H}$ & Steno-Mediterranean & & & \\
\hline Phalaris coerulescens Desf. & $\mathrm{H}$ & Steno-Mediterranean & & & \\
\hline Phalaris truncata Guss. & $\mathrm{H}$ & Euri-Mediterranean & & & \\
\hline Phleum pratense $\mathrm{L}$. & $\mathrm{H}$ & Circumboreal & & & F-B \\
\hline Phragmites australis (Cav.) Trin. & G & Cosmopolitan & & & hy \\
\hline Picris echioides $\mathrm{L}$. & $\mathrm{T}$ & Euri-Mediterranean & & & St \\
\hline Picris hieracioides L. & $\mathrm{H}$ & Circumboreal & & & St \\
\hline Pyrus pyraster Burgsd. & $\mathrm{P}$ & Eurasiatic & & & Q-F \\
\hline Pisum sativum $\mathrm{L}$. & $\mathrm{T}$ & Steno-Mediterranean & & & \\
\hline Plantago lanceolata $\mathrm{L}$. & $\mathrm{H}$ & Eurasiatic & & & M-A \\
\hline Роа аппиа $\mathrm{L}$. & $\mathrm{T}$ & Cosmopolitan & & & St \\
\hline Poa trivialis $\mathrm{L}$. & $\mathrm{H}$ & Eurasiatic & & & M-A \\
\hline Polypogon monspeliensis (L.) Desf. & $\mathrm{T}$ & Subtropical & & & \\
\hline Populus alba $\mathrm{L}$. & $P$ & Eurasiatic & & & hy \\
\hline Populus nigra $\mathrm{L}$. & $\mathrm{P}$ & Eurasiatic & & & hy \\
\hline Portulaca oleracea $\mathrm{L}$. & $\mathrm{T}$ & Cosmopolitan & & & St \\
\hline Potentilla reptans $\mathrm{L}$. & $\mathrm{H}$ & Eurasiatic & & & \\
\hline Prunus armeniaca $\mathrm{L}$. & $\mathrm{P}$ & Alien & hort. & cas & \\
\hline Prunus persica (L.) Batsch & $\mathrm{P}$ & Alien & hort. & cas & \\
\hline Prunus cerasifera Ehrh. & $\mathrm{P}$ & Alien & Eur, Asia & cas & \\
\hline Prunus spinosa $\mathrm{L}$. & $P$ & Eurasiatic & & & R-P \\
\hline Pteridium aquilinum (L.) Kuhn & G & Cosmopolitan & & & \\
\hline
\end{tabular}


Filibeck G., Cornelini P., Petrella P.

Tab. 1. - continued

\begin{tabular}{|c|c|c|c|c|c|}
\hline Species & $\begin{array}{l}\text { Life } \\
\text { form }\end{array}$ & $\begin{array}{l}\text { Geographic } \\
\text { element }\end{array}$ & $\begin{array}{l}\text { Origin } \\
\text { of aliens }\end{array}$ & $\begin{array}{l}\text { Status } \\
\text { of aliens } \\
\text { (Lazio) }\end{array}$ & $\begin{array}{l}\text { Vegeta- } \\
\text { tion } \\
\text { units }\end{array}$ \\
\hline Pulicaria dysenterica (L.) Bernh. & $\mathrm{H}$ & Euri-Mediterranean & & & \\
\hline Pyracantha coccinea Roemer & $\mathrm{P}$ & Steno-Mediterranean & & & R-P \\
\hline Pyrus pyraster Burgsd. & $P$ & Eurasiatic & & & Q-F \\
\hline Quercus robur $\mathrm{L}$. & $\mathrm{P}$ & Eurasiatic & & & hy \\
\hline Ranunculus bulbosus $\mathrm{L}$. & $\mathrm{H}$ & Eurasiatic & & & F-B \\
\hline Raphanus raphanistrum $\mathrm{L}$. & $\mathrm{T}$ & Euri-Mediterranean & & & St \\
\hline Rapistrum rugosum (L.) All. & $\mathrm{T}$ & Euri-Mediterranean & & & \\
\hline Reichardia picroides (L.) Roth & $\mathrm{H}$ & Steno-Mediterranean & & & T-Brom \\
\hline Reseda phyteuma $\mathrm{L}$. & $\mathrm{T}$ & Euri-Mediterranean & & & St \\
\hline Robinia pseudoacacia $\mathrm{L}$. & $P$ & Alien & N Amer & inv & \\
\hline Rosa canina $\mathrm{L}$. & $P$ & Eurasiatic & & & R-P \\
\hline Rosa sempervirens $\mathrm{L}$. & $P$ & Steno-Mediterranean & & & Qi \\
\hline intbl Rubia peregrina $\mathrm{L}$. & $P$ & Steno-Mediterranean & & & Qi \\
\hline Rubus ulmifolius Schott & $\mathrm{P}$ & Euri-Mediterranean & & & R-P \\
\hline Rumex conglomeratus Murray & $\mathrm{H}$ & Eurasiatic & & & \\
\hline Rumex crispus $\mathrm{L}$. & $\mathrm{H}$ & Cosmopolitan & & & \\
\hline Rumex obtusifolius L. & $\mathrm{H}$ & Eurasiatic & & & G-U \\
\hline Rumex pulcher $\mathrm{L}$. & $\mathrm{H}$ & Euri-Mediterranean & & & \\
\hline Salix alba $\mathrm{L}$. & $P$ & Eurasiatic & & & hy \\
\hline Salvia verbenaca $\mathrm{L}$. & $\mathrm{H}$ & Atlantic & & & \\
\hline Sambucus ebulus L. & G & Euri-Mediterranean & & & G-U \\
\hline Sanguisorba minor Scop. & $\mathrm{H}$ & Eurasiatic & & & F-B \\
\hline Saponaria officinalis $\mathrm{L}$. & $\mathrm{H}$ & Circumboreal & & & hy \\
\hline Scabiosa maritima $\mathrm{L}$. & $\mathrm{H}(\mathrm{b})$ & Steno-Mediterranean & & & F-B \\
\hline Scorpiurus muricatus $\mathrm{L}$. & $\mathrm{T}$ & Euri-Mediterranean & & & $\mathrm{Hg}$ \\
\hline Securigera securidaca (L.) Deg. et Doerfl. & $\mathrm{T}$ & Euri-Mediterranean & & & $\mathrm{Hg}$ \\
\hline Senecio vulgaris $\mathrm{L}$. & $\mathrm{T}$ & Euri-Mediterranean & & & St \\
\hline Setaria verticillata (L.) Beauv. & $\mathrm{T}$ & Subtropical & & & \\
\hline Setaria viridis (L.) Beauv. & $\mathrm{T}$ & Cosmopolitan & & & St \\
\hline Sherardia arvensis $\mathrm{L}$. & $\mathrm{T}$ & Euri-Mediterranean & & & St \\
\hline Silene alba (Miller) Krause & $\mathrm{H}(\mathrm{b})$ & Eurasiatic & & & $\mathrm{Av}$ \\
\hline Silene bellidifolia Juss. & $\mathrm{T}$ & Steno-Mediterranean & & & \\
\hline Silene latifolia Poiret & $\mathrm{H}(\mathrm{b})$ & Steno-Mediterranean & & & $\mathrm{G}-\mathrm{U}$ \\
\hline Silene vulgaris (Moench) Garcke & $\mathrm{H}$ & Eurasiatic & & & \\
\hline Sinapis arvensis $\mathrm{L}$. & $\mathrm{T}$ & Steno-Mediterranean & & & St \\
\hline Solanum nigrum $\mathrm{L}$. & $\mathrm{T}$ & Cosmopolitan & & & St \\
\hline Sonchus asper (L.) Hill & $\mathrm{T}$ & Eurasiatic & & & St \\
\hline Sonchus oleraceus L. & $\mathrm{T}$ & Eurasiatic & & & St \\
\hline Sonchus tenerrimus $\mathrm{L}$. & $\mathrm{T}$ & Steno-Mediterranean & & & St \\
\hline Sorghum halepense (L.) Pers. & G & Alien & Afr, Asia & inv & \\
\hline Spartium junceum L. & $P$ & Euri-Mediterranean & & & R-P \\
\hline Stachys ocymastrum (L.) Briq. & $\mathrm{T}$ & Steno-Mediterranean & & & \\
\hline Stellaria media (L.) Vill. & $\mathrm{T}$ & Cosmopolitan & & & St \\
\hline
\end{tabular}


Tab. 1. - continued

\begin{tabular}{|c|c|c|c|c|c|}
\hline Species & $\begin{array}{l}\text { Life } \\
\text { form }\end{array}$ & $\begin{array}{l}\text { Geographic } \\
\text { element }\end{array}$ & $\begin{array}{l}\text { Origin } \\
\text { of aliens }\end{array}$ & $\begin{array}{l}\text { Status } \\
\text { of aliens } \\
\text { (Lazio) }\end{array}$ & $\begin{array}{l}\text { Vegeta- } \\
\text { tion } \\
\text { units }\end{array}$ \\
\hline Sylibum marianum (L.) Gaertner & $\mathrm{H}(\mathrm{b})$ & Medit.-Turanic & & & G-U \\
\hline Taraxacum officinale Weber & $\mathrm{H}$ & Circumboreal & & & M-A \\
\hline Thymus vulgaris $\mathrm{L}$. & Ch & Steno-Mediterranean & & & $\mathrm{C}-\mathrm{M}$ \\
\hline Tolpis virgata (Desf.) Bertol. & $\mathrm{H}$ & Steno-Mediterranean & & & St \\
\hline Torilis arvensis (Hudson) Link & $\mathrm{T}$ & Cosmopolitan & & & \\
\hline Tragopogon porrifolius $\mathrm{L}$. & $\mathrm{H}(\mathrm{b})$ & Euri-Mediterranean & & & $\mathrm{Av}$ \\
\hline Trifolium angustifolium $\mathrm{L}$. & $\mathrm{T}$ & Euri-Mediterranean & & & T-Brom \\
\hline Trifolium arvense $\mathrm{L}$. & $\mathrm{T}$ & Eurasiatic & & & \\
\hline Trifolium campestre Schreber & $\mathrm{T}$ & Eurasiatic & & & St \\
\hline Trifolium fragiferum $\mathrm{L}$. & $\mathrm{H}$ & Eurasiatic & & & \\
\hline Trifolium ochroleucum Hudson & $\mathrm{H}$ & Eurasiatic & & & F-B \\
\hline Trifolium pratense $\mathrm{L}$. & $\mathrm{H}$ & Circumboreal & & & M-A \\
\hline Trifolium subterraneum $\mathrm{L}$. & $\mathrm{T}$ & Euri-Mediterranean & & & \\
\hline Trisetaria panicea (Lam.) Maire & $\mathrm{T}$ & Steno-Mediterranean & & & T-Brom \\
\hline Tussilago farfara $\mathrm{L}$. & G & Eurasiatic & & & $\mathrm{Av}$ \\
\hline Typha latifolia $\mathrm{L}$. & G & Cosmopolitan & & & hy \\
\hline Ulmus minor Miller & $\mathrm{P}$ & Eurasiatic & & & Q-F \\
\hline Urospermum dalechampii (L.) Schmidt & $\mathrm{H}$ & Euri-Mediterranean & & & T-Brom \\
\hline Urtica dioica $\mathrm{L}$. & $\mathrm{H}$ & Cosmopolitan & & & G-U \\
\hline Urtica membranacea Poiret & $\mathrm{T}$ & Steno-Mediterranean & & & St \\
\hline Valerianella eriocarpa Desv. & $\mathrm{T}$ & Steno-Mediterranean & & & St \\
\hline Verbascum blattaria $\mathrm{L}$. & $\mathrm{H}(\mathrm{b})$ & Eurasiatic & & & Av \\
\hline Verbascum densiflorum Bertol. & $\mathrm{H}(\mathrm{b})$ & Euri-Mediterranean & & & Av \\
\hline Verbascum pulverulentum Vill. & $\mathrm{H}(\mathrm{b})$ & Eurasiatic & & & Av \\
\hline Verbascum sinuatum $\mathrm{L}$. & $\mathrm{H}(\mathrm{b})$ & Euri-Mediterranean & & & Av \\
\hline Verbena officinalis $\mathrm{L}$. & $\mathrm{H}$ & Eurasiatic & & & St \\
\hline Veronica arvensis L. & $\mathrm{T}$ & Eurasiatic & & & St \\
\hline Veronica cymbalaria Bodard & $\mathrm{T}$ & Euri-Mediterranean & & & \\
\hline Veronica hederifolia $\mathrm{L}$. & $\mathrm{T}$ & Eurasiatic & & & St \\
\hline Veronica persica Poiret & $\mathrm{T}$ & Alien & Asia & inv & \\
\hline Vicia bithynica (L.) L. & $\mathrm{T}$ & Euri-Mediterranean & & & \\
\hline Vicia hirsuta (L.) Gray & $\mathrm{T}$ & Eurasiatic & & & \\
\hline Vicia hybrida $\mathrm{L}$. & $\mathrm{T}$ & Euri-Mediterranean & & & \\
\hline Vicia lutea $\mathrm{L}$. & $\mathrm{T}$ & Euri-Mediterranean & & & St \\
\hline Vicia sativa $\mathrm{L}$. & $\mathrm{T}$ & Medit.-Turanic & & & St \\
\hline Vicia villosa Roth & $\mathrm{T}$ & Euri-Mediterranean & & & T-Brom \\
\hline Vitis vinifera $\mathrm{L}$. & $\mathrm{P}$ & Alien & hort. & cas & \\
\hline Vulpia ciliata (Danth.) Link & $\mathrm{T}$ & Euri-Mediterranean & & & T-Brom \\
\hline Vulpia ligustica (All.) Link & $\mathrm{T}$ & Steno-Mediterranean & & & T-Brom \\
\hline Zea mays $\mathrm{L}$. & $\mathrm{T}$ & Alien & hort. & cas & \\
\hline
\end{tabular}


embankment communities are either biennial or rosulate plants: typical examples include Daucus carota, Malva sylvestris, Plantago lanceolata, Picris hieracioides, and four different Verbascum species. Phanerophytes featured $13 \%$ of the recorded flora (37 species).

The geographic element with the highest number of species (Fig. 2) was the Eurasiatic (ca. 30\%), followed by euri-Mediterranean and steno-Mediterranean elements - however, the sum of these latter (and of the Mediterranean-Turanic element) is ca $44 \%$ of the recorded flora.

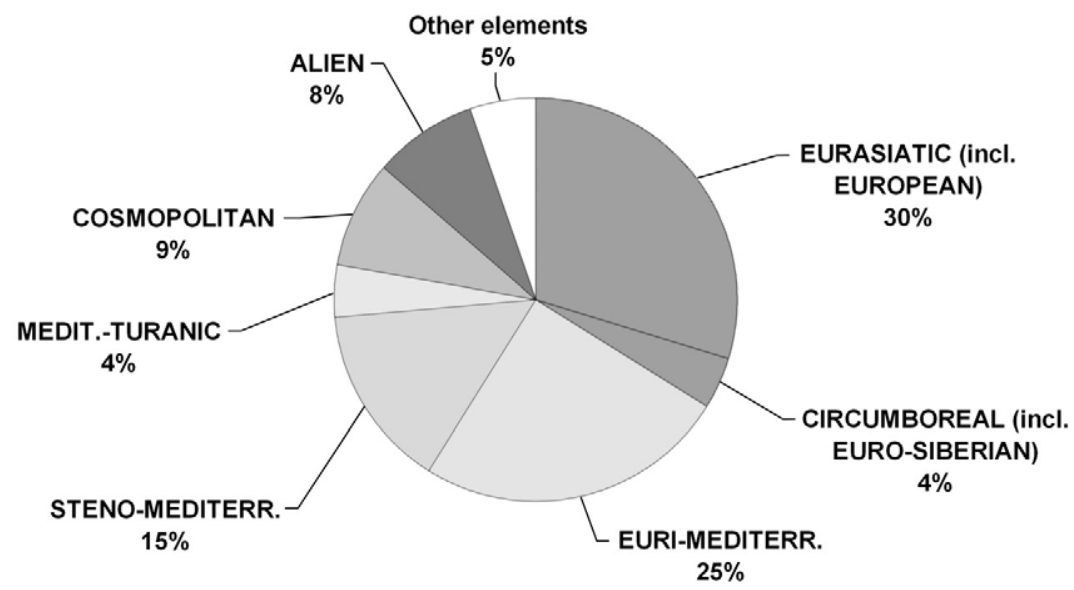

Fig. 2. Geographic elements in the flora of the railway segment. For explanations see table 1.

Alien species (including taxa that originated as cultivated plants) accounted for $8.4 \%$. Alien species classified as »invasive « in Lazio were $4 \%$ of the recorded flora.

In the phytosociological spectrum (Fig. 3), the largest element (75 species, i.e. 26\%) was made up by species from Stellarietea mediae, but 20 out of these 75 species belong to the Thero-Brometalia order. Species from Festuco-Brometea are also represented in the embankment flora (16 species, 5.6\%), while the Molinio-Arrhenateretea element is poorer ( 8 species). Artemisietea vulgaris class is represented by 19 species (6.6\%), and Galio-Urticetea features only 10 species $(3.5 \%)$. A total of 26 species $(9.1 \%)$ belongs to the floristic assemblage of forest or forest-edge communities. Finally, $6.3 \%$ of the flora is made up of species from hygrophilous syntaxa.

\section{Discussion}

The total richness found (with more than 200 taxa recorded in the first 1,000 m) is considerably high compared to some north European studies on railways (cf. e.g. MESSENGER 1968, NiEMI 1969, LeJMBACH et al. 1975), but is consistent with the high floristic richness of Mediterranean landscapes (the Lazio region has 3,228 taxa: CoNTI et al. 2005) and with species-area patterns known for central Italy (CHIARUCCI et al. 2012). In the family spectrum, the richest families recorded from the embankment are the same as in the whole Italian flora (ABBATE et al. 2007), however in the embankment flora there is an anomalous proportion of Scrophulariaceae, Lamiaceae and Boraginaceae, due to the disturbance regime of the railway sides, which promotes the diversity of ruderal and/or biennial species 


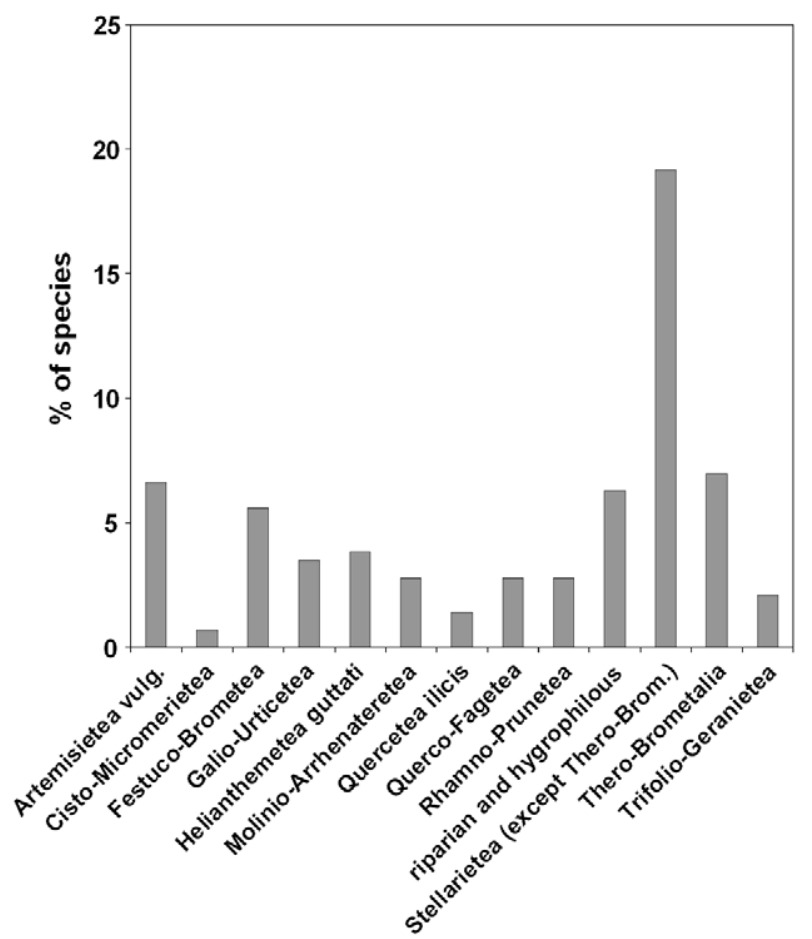

Fig. 3. Proportion of character species of different syntaxa in the flora of the railway segment.

- particularly well represented in these families. The most common annuals in the embankment flora included Avena barbata, Bromus madritensis, Papaver rhoeas and Sherardia arvensis (CORNELINI 1994b).

The life-form distribution, despite the artificial and recent origin of the study site, keeps a strong similarity with the spectrum of the regional pool (Fig. 4). Annuals feature a higher proportion than in the flora of Lazio ( $38 \%$ vs. $33 \%$ ), as expected due to three co-occurring factors: the warm climate belt where the study area is located; the dry topsoil of the embankment; and the disturbance caused by mowing of the embankment slopes. The proportion of therophytes found here is much lower than that recorded in a railway station in Rome by CORNELINI and PETRELla (1996), where 50\% of a 266-species flora was made up of annuals - however, the two study sites are not comparable as the railway station includes large paved areas and/or highly disturbed habitats, and it is surrounded by a completely urbanized district. Perennial herbs have a slightly higher proportion in the embankment assemblage than in the regional flora. The richness of biennial and rosulate Hemicryptophytes is well known to be connected to the features of railway verges (TINNER and SCHUMACHER 2004, NowińsKA and CZARNA 2008): traits such as buds very close to the ground, long taproots and flat rosettes allow survival in spite of frequent mechanical mowing. Geophytes, although with a lower percentage than in the regional flora, are well-represented, since these plants are favorably selected by an intermediate intensity of mechanical disturbance - as already observed e.g. in the archaeological areas in downtown Rome (CELESTI-GRAPOW et al. 1989, Celesti-Grapow 1995). Among the Geophytes found in the embankment flora, 


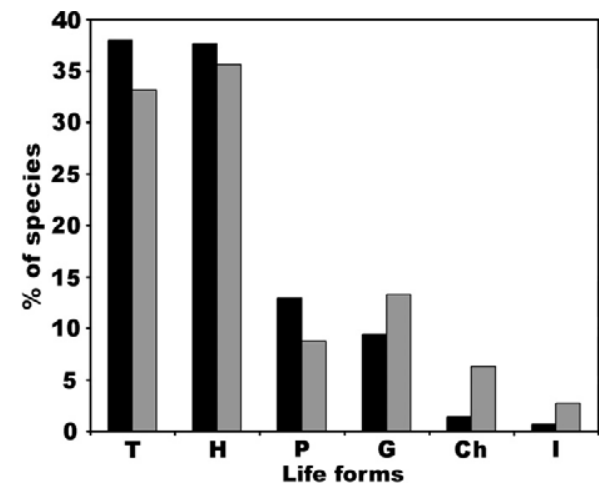

Fig. 4. Comparison between the life-form distribution in the embankment flora (black columns) and in the flora of Lazio region (grey columns). For legend of Life forms, see table 1.

the most noteworthy include an orchid, Ophrys apifera, and Cyclamen hederifolium, a species usually bound to the shady understorey of sub-Mediterranean woods. On the other hand, the high proportion of Phanerophytes highlights the role played by the railway sides as a substitute habitat for woody species, within an intensively farmed landscape devoid of suitable microsites such as hedgerows or dry stone walls. In fact, among the Phanerophytes recorded on the rail embankment, there are species from different vegetation series of the toposequence of the Tiber valley, and from different seral stages of each vegetation series: Populus alba and Salix alba are trees from the river-side forest vegetation (CESCHIN and SALERNO 2008); Fraxinus oxycarpa and Quercus robur are trees which potentially dominate the alluvial, hygro-mesophytic plains (BLASI et al. 2010); Cornus sanguinea and Euonymus europaeus are shrubby species from the wood edges of the alluvial plain forest (CESCHIN and SALERNO 2008); Fraxinus ornus and Pyrus pyraster are small trees of the thermophylous Quercus cerris woods of the hill slopes (BLASI et al. 2004); Cercis siliquastrum, Crataegus monogyna and Pyracantha coccinea are found in the wood edges and shrubby seral stages of the Q.cerris forests (BLASI et al. 2004); Celtis australis and Ficus carica are often found on the pyroclastic cliffs and boulders (SCOPPOLA and FILIBECK 2008).

As for the geographic element distribution (Fig. 2), the Mediterranean element (in a wide sense) prevails in the studied railway side flora (ca. 44\%), suggesting that the Mediterranean climate acts as a strong ecological filter, so that a marked link with the regional species pool is preserved even in a completely artificial habitat.

The richness of alien species found in the study site $(8.4 \%)$ is relatively low for an artificial, disturbed habitat, considering that in the chorological spectrum of the whole flora of the Lazio region alien units reach $10.4 \%$ (CONTI et al. 2005). Studies from central European railway floras found percentages of alien species up to 36\% (Fig. 5) (LEJMBACH et al. 1975, TinNer and SCHUMACHER 2004, NowińsKa and CZARna 2008, WARCHOLIŃSKA and SuWARA-SZMigielSKa 2009). However, these studies are not fully comparable with the present work, as they include (or are limited to) stations and/or urban habitats.

Alien therophytes are a group that in central and north Europe is often reported to play a significant role in the flora of railway areas (e.g. NiEMI 1969, BRANDES 1983, TINNER and SCHUMACHER 2004, GALERA et al. 2011). For instance, in a floristic study on railway lines and 


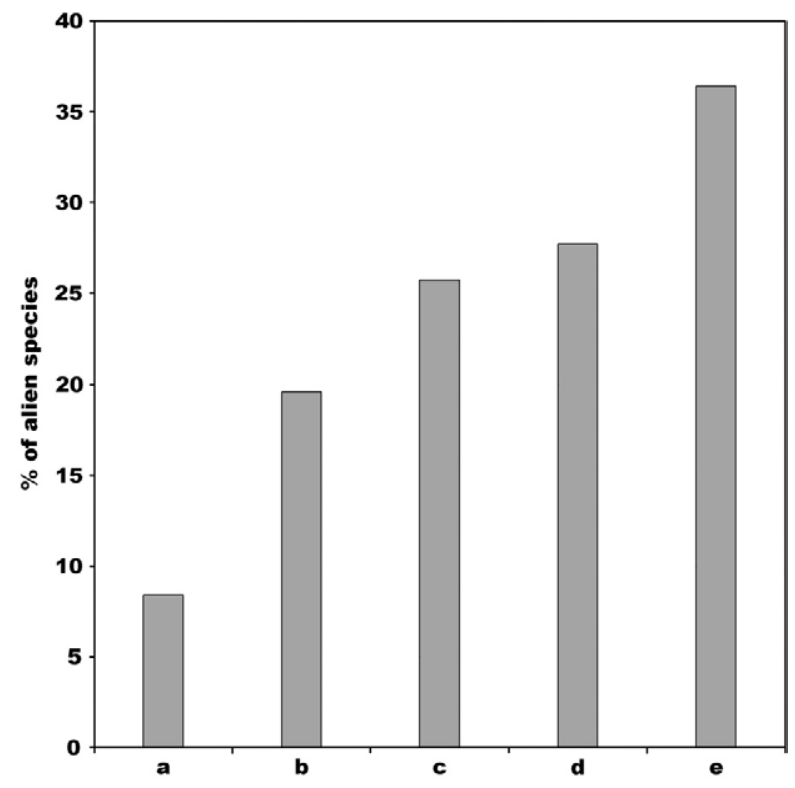

Fig. 5. Proportion of alien species recorded in various railway floras: a) present study (8.4\%); b) railway stations of NE-Switzerland (19.6\%) (TINNER and Schumacher 2004); c) Powodowo station, W-Poland (25.7\%) (NowIŃSKA and CZARNA 2008); d) railway lines and stations of Pomerania, N-Poland (27.7\%) (LEJMBACH et al. 1975); e) urban railways and stations in Pabianice, C-Poland (36.4\%) (WARCHOLIŃSKA and SUWARA-SZMIGIELSKA 2009).

stations of Pomerania (LEJMBACH et al. 1975), 22.3\% of the total railway flora was made up of alien therophytes (including alien biennial-hemicryptophytes). This group, by contrast, had a very low diversity in our survey: only 6 species $(2.1 \%)$ of exotic annuals were found (Amaranthus retroflexus, Conyza bonariensis, Conyza canadensis, Euphorbia prostrata, Veronica persica, Zea mays). Although our result could be influenced by the fact that in the present study the ballast was not surveyed, it has already been underlined that in the floras of Mediterranean cities (e.g. CELESTI-GRAPOw 1995, CELESTI-GrAPOw et al. 1997) alien annuals are not as characteristic as they are in central European urban environments. This is because in the Temperate biome artificial habitats perform as extrazonal, dry and warm micro-environments: the local species are less adapted to this niche, which is more competitively exploited by aliens from warmer countries. In the Mediterranean biome, instead, xeric conditions are widespread and thus railway grounds do not perform as especially favorable sites for therophytes (BRANDES 1993: 441).

In the phytosociological spectrum (Fig. 3), the richest group (26\%) is made up by species recognized as characteristic taxa of Stellarietea mediae (or of its subordinate orders), i.e. the widely-distributed class of the annual, ruderal communities (according to GRIME et al. 1988), bound to highly disturbed or anthropogenic soils (e.g. MucinA et al. 1993). However, a large share of the Stellarietea species recorded in the embankment flora is accounted for by taxa belonging to the order Thero-Brometalia (=Brometalia rubenti-tectorum) and in particular to the Echio-Galactition alliance, i.e. to the Mediterranean, sub-nitrophilous and medium-sized annual vegetation communities of fallow land, minor-road sides and other edge ha- 
bitats (IzCo 1977, GigAnTE and VenANZONi 2007). All these species (e.g. Avena barbata, Bromus hordeaceus, Dasypyrum villosum, Lotus ornithopodioides, Medicago orbicularis, Reichardia picroides), although with a partially ruderal character, have a decidedly xerophilous ecology and a strictly Mediterranean range - so that many of them were until recently classified among the characteristic species of the natural and semi-natural Mediterranean annual grasslands of Helianthemetea guttati (=Thero-Brachypodietea) (e.g. RIVAS-MARTINEZ 1977, FANELLI and LUCCHESE 1998). Further, even this latter class, interpreted in a narrower sense (i.e. without the above mentioned sub-nitrophilous species), is represented in our survey by a significant group of species (e.g. Briza maxima, Blackstonia perfoliata, Hypochoeris achyrophorus, Scorpiurus muricatus). On the other hand, the taxa from Artemisietea vulgaris [i.e. the Euro-Siberian communities of perennial, nitrophilous to sub-nitrophilous herbs and grasses with an intermediate C-R behaviour (according to GRIME et al., 1988), usually typical of road- and railway-sides (MucINA et al. 1993)] are not particularly numerous in the embankment (19 species, 6.6\%), as their functional traits are scarcely adapted to the Mediterranean climate. The same holds for the species from Galio-Urticetea (wood- and road-fringe communities of nitrophilous, tall herbs with a C-R or C strategy) (MucinA et al. 1993), featuring only 10 species $(3.5 \%)$ and from Molinio-Arrhenateretea (nitrogen-rich and mesophytic anthropogenic hay-meadows of the temperate region) (8 species). We defined as forest species $(9.1 \%)$ the taxa known as characteristic species of the following classes (or subordinate syntaxa): Quercetea ilicis (Mediterranean evergreen woods and maquis: e.g. Rubia peregrina, Asparagus acutifolius); Querco-Fagetea (European deciduous forests namely, in the surroundings of the study area, sub-Mediterranean thermophilous deciduous forests of the order Quercetalia pubescenti-petraeae: e.g. Cyclamen hederifolium, Cercis siliquastrum, Fraxinus ornus); Rhamno-Prunetea (deciduous forest-edge shrublands: e.g. Crataegus monogyna, Prunus spinosa); Trifolio-Geranietea (communities of the partially shaded, herbaceous fringes at the edge of deciduous woodlands; e.g. Agrimonia eupatoria, Holcus lanatus, Lathyrus sylvestris, Origanum vulgare, Sanguisorba minor). Species from (woody or herbaceous) hygrophilous syntaxa (Lemnetea, Phragmito-Magnocaricetea, Salicetea purpureae, Populetalia albae) are also found in a noteworthy proportion $(6.3 \%)$ : these plants (e.g. Alisma plantago-aquatica, Carex otrubae, Lemna minor, Typha latifolia) are common within the surveyed railway sides because of the numerous drainage ditches at the foot of the embankment - and because the vicinity of the river Tiber is a source of propagules (cf. CESCHIN and SALERNO 2008).

Thus, the spectrum of the original plant communities of the recorded species shows that the flora of the studied railway section is only partially »ruderalized «: on the contrary, it seems to keep a strong link with the floristic composition of the plant communities of its biogeographic context. This confirms the suggestion of a comparative study on urban floras of Italy (CELESTI-GRAPOW et al. 1997), where the floristic assemblage in cities belonging to the Mediterranean sector of the country was found to have a high degree of similarity with the floristic composition of the surrounding (semi-)natural landscapes, while such a correlation was much weaker in cities of the temperate sector of Italy.

Further, the different ecological elements that can be traced in the recorded flora underline the extremely high heterogeneity of micro-habitats within the railway sides, and hence their potential importance for biodiversity: the co-occurrence in the surveyed flora of, for 
instance, hygrophytes such as Salix alba and xerophytes such as Micromeria graeca highlights the very steep gradient taking place within the 20-m wide embankment.

In contrast to many central and north European studies (RANTA 2008 with references), no National/Regional Red List taxa or other particularly rare species were found. However, in temperate Europe rail verges have a high percentage of rare/endangered species because they provide a much drier micro-habitat than the surrounding landscape (BRANDES 1993), and thus are capable of hosting extrazonal Mediterranean taxa and other thermophilous plants (which include many of the Red List species in central European countries). As for boreal Europe, semi-natural grasslands (meadows and pastures which have originated from grazing and hay-making on natural grasslands and in woodlands) account for up to $25 \%$ of the endangered plant species, and are a particularly threatened habitat-type because of the cessation of grazing and mowing and their conversion to arable and afforested land (TIKKA et al. 2000, 2001): for these reasons, mowed road- and rail-verges are nowadays among the largest surviving areas of grassland habitats in north Europe. This is not applicable in Mediterranean Europe, where dry habitats are the rule rather than the exception, and where grasslands are still widely represented (BLASI et al. 2007, 2010). Thus, in south Europe road and rail verges are not necessarily valuable habitats in every landscape context, and probably do not play a significant role for endangered species. However, in the highly transformed landscape of the present study, the railway sides provided a decidedly less disturbed habitat (although on a completely artificial soil), containing a significant sample of the floristic assemblages of many different natural and semi-natural communities of a wider area - thus probably playing an important role, at a local scale, in maintaining diversity patterns and in providing corridors through an unfavorable landscape matrix.

The present work was the first study carried out in Italy - and probably in Mediterranean Europe - on the floristic composition of a long railway segment beyond rail stations or urban areas, and perhaps the first study ever performed in Europe on a high-speed line.

The floristic assemblage recorded in the studied rail embankment suggests that the ecological filter operated by the Mediterranean macroclimate prevents both complete »ruderalization« and »floristic homogenization « in the flora of even a highly artificial habitat - by contrast to what is observed in Temperate Europe and north America, where floras of anthropogenic habitats from different countries were found to be more similar to each other than to the surrounding (semi-) natural habitats (WITTIG and BECKER 2010).

Further studies gathering a large amount of floristic data from railway grounds and other artificial habitats in south Europe are needed to confirm what is suggested by the present work. This is relevant also for planning restoration practices of artificial substrata (embankments, road cuts, etc.), since it means that in the Mediterranean region the climate prevails over the edaphic filter. Thus, the surrounding natural communities can provide a good reference list for choosing the species to be used for revegetation practices, and, as tested by recent studies in Mediterranean areas (Bochet et al. 2007, Mola et al. 2011), it is even possible to rely only on natural colonization processes.

Further, as already found in central and north Europe (TIKKA et al. 2000; 2001, TINNER and Schumacher 2004, RANTA 2008, Lososova and LANikova 2010), in the Mediterranean region too railway sides can act as a substitute habitat for species from natural and semi-natural communities. However, the ecological role of railway sides in the temperate 
and boreal regions - where road and railway verges are among the few surviving examples of dry and mowed habitats - is quite different compared to the Mediterranean countries.

Finally, it is to be stressed that the maintenance regime has a crucial role: at the time of our survey, rail-side management consisted only of infrequent mechanical cutting, which allowed the development of a complex pattern of many different seral stages (including shrub communities), thus promoting floristic diversity.

\section{References}

Abbate, G., Alessandrini, A., Conti, F., 2007: Vascular plants. In: Blasi, C., Boitani, L., La Posta, S., Manes, F., Marchetti, M. (eds.), Biodiversity in Italy, 149-161. Palombi Editori, Roma.

Biondi, E., BAllelli, S., AllegrezZA, M., ZucCAREllo, V., 1995: La vegetazione dell' ordine Brometalia erecti Br.-Bl. 1936 nell' Appennino (Italia). Fitosociologia 30, 3-45.

Blasi, C., 1994: Fitoclimatologia del Lazio. Fitosociologia 27, 151-175.

Blasi, C., Di Pietro, R., Filesi, L., 2004: Syntaxonomical revision of Quercetalia pubescenti-petraeae in the Italian peninsula. Fitosociologia 41, 87-164.

Blasi, C., Boitani, L., La Posta, S., Manes, F., Marchetti, M. (eds.), 2007: Biodiversity in Italy. Palombi Editori, Roma.

Blasi, C., Di Pietro, R., Filibeck, G., Filesi, L., Ercole, S., Rosati, L., 2010: Le serie di vegetazione della regione Lazio. In: BLASI, C. (ed.), La vegetazione d'Italia, 281-309. Palombi Editori, Roma.

Bochet, E., Garcia-Fayos, P., Tormo, J., 2007: Road slope revegetation in semiarid Mediterranean environments. Part I: seed dispersal and spontaneous colonization. Restoration Ecology 15, 88-96.

BRANDES, D., 1983: Flora und Vegetation der Bahnhöfe Mitteleuropas. Phytocoenologia 11, 31-115.

BRANDES, D.,1992: Spontane Vegetation von ligurischen Küstenorten. Braun-Blanquetia 3, 229-240.

BRANDES, D., 1993: Eisenbahnanlagen als Untersuchungsgegenstand der Geobotanik. Tuexenia $13,415-444$.

BRANDES, D., 2008: Bibliographie zur Eisenbahnvegetation. Technische Universität Carolo-Wilhelmina, Universitätbibliothek, Braunschweig. URL: http://www.digibib.tu-bs.de/ ?docid=00021885 (accessed January 13, 2012).

CACCIATO, A., 1952: La vegetazione antropocora dello scalo ferroviario Ostiense di Roma. Nuovo Giornale Botanico Italiano 59, 119-143.

CElesti-Grapow, L., 1995: Atlante della flora di Roma. Argos, Roma..

Celesti-Grapow, L., Blasi, C., Andreis, C., Biondi, E., Raimondo, F. M., Mossa, L., 1997: Studio comparativo sulla flora urbana in Italia. Giornale Botanico Italiano 130, 779-793.

Celesti-Grapow, L., Pretto, F., Carli, E., Blasi, C., 2010: Flora vascolare alloctona e invasiva delle regioni d'Italia. Casa Editrice Università La Sapienza, Roma.

Ceschin, S., SAlerno, G., 2008: La vegetazione del basso corso del Fiume Tevere e dei suoi affluenti (Lazio, Italia). Fitosociologia 45, 39-74. 
Chiarucci, A., Bacaro, G., Filibeck, G., Landi, S., Maccherini S., Scoppola A., 2012: Scale dependence of plant species richness in a network of protected areas. Biodiversity and Conservation 21, 503-516.

Christen, D., Matlack, G., 2006: The role of roadsides in plant invasions: a demographic approach. Conservation Biology 20, 385-391.

Conti, F., Abbate, G., Alessandrini, A., Blasi, C., 2005: An annotated checklist of the Italian vascular flora. Palombi, Roma.

CORNELINI, P.,1994a: Evoluzione della vegetazione erbacea dei rilevati ferroviari. In: FERRARI, I., MANes, F., Biondi, E. (eds.), Alterazioni ambientali ed effetti sulle piante, 261-279. Edagricole, Bologna.

CoRnElini, P., 1994b: Valore e potenzialità naturalistiche delle scarpate ferroviarie. Ingegneria Ferroviaria 3, 1-7.

Cornelini, P., Petrella, P., 1996: La flora della stazione di Roma Ostiense: variazioni e confronti con il censimento di Cacciato (1952). Annali di Botanica (Roma) 52 (suppl. 11), 457-478.

Cornelini, P., Petrella, P., 1997: Indagini floristiche negli impianti ferroviari di Roma. Ingegneria Ferroviaria 3, 110-116.

Cornelini, P., Filesi, L., Paolella, A., Blasi, C., 1990: Indagini e considerazioni per il recupero delle aree ipostradali libere. Verde Ambiente 2, 31-41.

FANELLI, G., LuCCHESE, F., 1998: The status of Brometalia rubenti-tectorum communities from the Mediterranean area in different syntaxonomical schemes. Rendiconti Fisici Accademia dei Lincei 9, 241-255.

Filibeck, G., Scoppola, A., 2011: Two Mediterranean landscape types and their interface as a case study for »landscape red-listing «. Fitosociologia 48 (suppl. 1), 119-125.

FISCHER, J., LINDENMAYER, D. B., 2007: Landscape modification and habitat fragmentation: A synthesis. Global Ecology and Biogeography 16, 265-280.

Galera, H., Sudnik-Wójcikowska, B., Wierbzbicka, M., WiŁKomirski, B., 2011: Encroachment of forest species into operating and abandoned railway areas in north-eastern Poland. Plant Biosystems 145, 23-36.

Gigante, D., Venanzoni, R., 2007: Some remarks about the annual sub-nitrophilous vegetation of Thero-Brometalia in Umbria (Central Italy). Lazaroa 28, 15-34.

Grime, J. P., Hogdson, J. G., Hunt, R., 1988: Comparative plant ecology. Unwyn Nyman, Boston, Sidney, Wellington.

IzCO, J., 1977: Revision sintetica de los pastizales del suborden Bromenalia rubenti-tectori. Colloques Phytosociologiques 6, 37-52.

Koster, A., 1987: The flora of Dutch railways (in Dutch). Ministerie van Landbouw en Visserij, The Hague.

Lejmbach, B., Rurka, Z., Siedlecka, B., Sijka, J., 1975: The flora of railway tracks of eastern Pomerania coast (in Polish). Fragmenta Floristica et Geobotanica 21, 53-66.

Lososova, Z., LANIKOVA, D., 2010: Differences in trait compositions between rocky natural and artificial habitats. Journal of Vegetation Science 21, 520-530.

Messenger, K. G., 1968: A railway flora of Rutland. Proceedings of the Botanical Society of the British Isles 7, 325-344. 
Mola, I., Jimenez, M. D., Lopez-Jimenez, N., Casado, M. A., Balaguer, L., 2011: Roadside reclamation outside the revegetation season: management options under schedule pressure. Restoration Ecology 19, 83-92.

Mucina, L., GrabHerr, G., Ellmauer, T., 1993: Die Pflanzengesellschaften Österreichs. I. Antropogene Vegetation. G. Fischer Verlag, Stuttgart.

MüHlenbaCH, V., 1979: Contributions to the synanthropic (adventive) flora of the railroads in St. Louis, Missouri, USA. Annals of the Missouri Botanical Garden 66, 1-108.

NIEMI, A., 1969: On the railway vegetation and flora between Esbo and Ingå, S. Finland. Acta Botanica Fennica 83, 1-28.

NowiŃSKA, R., CZARNA, A., 2008: Impact of railway facility operation on floral growth in a Powodowo, the Region of Wielkopolska. Polish Journal Environmental Studies 17, 613-621.

OBERDORFER, E., 1992: Süddeutsche Pflanzengesellschaften. 3. Auflage. G. Fischer Verlag, Stuttgart.

PARR, T. W., WAY, J. M., 1988: Management of roadside vegetation: The long-term effects of cutting. Journal Applied Ecology 25, 1073-1087.

PignatTI, S., 1982: Flora d'Italia. Edagricole, Bologna.

RANTA, P., 2008: The importance of traffic corridors as urban habitats for plant in Finland. Urban Ecosystems 11, 149-159.

Rivas-MARTineZ, S., 1977: Sur la syntaxonomie des pelouses therophytiques de l'Europe occidentale. Colloques phytosociologiques 6, 55-69.

SARGENT, C., 1984.: Britain's railway vegetation. Cambridge, Institute of Terrestrial Ecology.

Schaffers, A. P., SiKorA, K. V., 2002: Synecology of species-rich plant communities on roadside verges in the Netherlands. Phytocoenologia 32, 29-83

Schoenenberger, N., Druart, P., Giorgetti Franscini, P., 2002: Note floristiche ticinesi: la flora della rete ferroviaria con particolare attenzione alle specie avventizie. Parte I. Bollettino Società Ticinese Scienze Naturali 90, 127-138.

Scoppola, A., Filibeck, G., 2008: Il paesaggio vegetale del Parco Regionale Marturanum. Parco Regionale Marturanum, Barbarano Romano.

Tikka, P. M., Koski, P. S., Kivelë, R. A., Kuitunen, M. T., 2000: Can grassland communities be preserved on road and railway verges? Applied Vegetation Science 3, 25-32.

TikKa, P. M., Högmander, H., Koski, P. S., 2001: Road and railway verges serve as dispersal corridors for grassland plants. Landscape Ecology 16, 659-666.

Tinner, U., Schumacher, H., 2004: Flora auf Bahnhöfen der Nordostschweiz. Botanica Helvetica 114, 109-125.

TRET'YAKOVA, A. S., 2010: The role of railroads in the formation of synanthropic flora in the Middle Urals. Russian Journal of Ecology 41, 123-128.

WarcholińsKa, A. U., SuwARA-SzmigielsKa, S., 2009: The vascular flora of the railway grounds of the Pabianice town. Folia Biologica et Oecologica 5, 21-41.

WitTIG, R., 2002: Ferns in a new role as a frequent constituent of railway flora in central Europe. Flora 197, 341-350.

WitTig, R., BeckeR, U., 2010: The spontaneous flora around street trees in cities - A striking example for the worldwide homogenization of the flora of urban habitats. Flora 205, 704-709. 